\title{
P 169 CAN ELECTRONIC EVALUATION OF EDUCATION DEMONSTRATE BENEFIT TO PRACTICE?
}

\author{
Laura Myers, Mary Mahoney. St Catherine's Hospice, Crawley, UK
}

10.1136/bmjspcare-2014-000654.210

Background St Catherine's hospice has designed a new organisational strategy, which includes reaching more people who are dying and their families, regardless of their illness. The success of such an endeavour is dependent on knowledgeable and confident clinical staff. A pilot electronic evaluation project has been established and is beginning to produce data showing the effect of education on practice.

Method Electronic links to questionnaires were sent to course participants immediately before and after attending study days and three months later. Core data comprised self-reported ratings of understanding and confidence based on learning outcomes. Additional pre-course data established the rationale for attendance and expected learning. Post-course data identified learning and how it would be used in practice, as well as feedback on the content, delivery, and pitch of individual sessions. The three month follow up questionnaire provided participants with the opportunity for reflection on their learning and asked for examples of its use in practice.

Results Completion of the forms was inconsistent; some participants finding the process easy, while others were hampered by their inability to use or access computers. A great deal of data was generated, including graphs showing enhanced self-reported post-course levels of confidence and understanding, qualitative comments, and use of learning in practice. Three types of reports were created: one for educators, containing all data and used for course review; the second for individual speakers, providing feedback on their sessions; and a third for commissioners to demonstrate perceived learning and application to clinical work. The project brought about a structure for gathering evaluation data and producing reports that are clear, concise and illustrate the difference education makes to practice. The design and implementation of this electronic process was initially resource intensive and now provides a structured method for data collection and analysis.

Conclusion Electronic evaluation of hospice education provides clearer data that learning benefits practice. 